\title{
A Note on Essential Soft Submodules
}

\author{
Bijan Davvaz ${ }^{1}$, Jing $\mathrm{Ma}^{2}$, and Chengxiz Sun ${ }^{3}$ \\ ${ }^{1}$ Department of Mathematics, Yazd University, Iran \\ ${ }^{2}$ Department of Mathematics, Jilin University, Changchun China \\ ${ }^{3}$ United Experimental School of AHSJU and Livon, Changchun, China
}

\section{]jfis}

\begin{abstract}
In this paper, we investigate the property of essential soft submodule and give an equivalent condition of essential soft submodule. Then the concept of the socle of a soft module is introduced, basic properties of such soft submodules are investigated, several equivalent definitions of the socle and the radical of a soft module are given respectively. Also, examples of these related soft submodules are given.
\end{abstract}

Keywords: Soft module, Essential soft submodule, Socle, Radical

\section{Introduction}

Many practical problems, in economics, engineering, environment, social science, medical science etc., with uncertainty as part of the data are not effectively dealt with by classical methods. Some of these difficulties is due to uncertainties and the inadequacy of the theories of parameterization tools. In 1999, Molodtsov [1] initiated the notion of soft sets as a general mathematical framework for dealing with uncertainty.

In 2003, Maji et al. [2] studied the operation on soft sets. In 2007, Aktas and Cagman [3] defined soft groups and compared soft sets with rough sets. In 2008, Feng et al. [4] introduced the notions of soft semirings and soft ideals, and then Acar et al. [5] introduced initial concepts of soft rings in 2010. In 2009, Irfan et al. [6] defined a new operation on soft sets.

In 2008, Sun et al. [7] defined soft modules and investigated their basic properties. In 2011, Atagun and Sezgin [8] studied soft substructures of rings and modules; Xiao [9] proved the isomorphism theorems of soft modules and studied the exact sequence of soft modules; Gunduz and Bayramov [10] introduced the concept of intuitionistic fuzzy soft module and some operations on intuitionistic fuzzy soft modules. In 2012, Yin et al. [11] discussed the operation properties and algebraic structure of intuitionistic fuzzy soft sets. In 2013, Xiang [12] studied soft modules; Ozturk et al. [13] investigated the inverse and direct systems of soft modules; Turkmen and Pancar [14] introduce the notions of sum and direct sum of soft submodules, small soft submodules and the radical of a soft module, and discussed the relation of the radical of a soft module with its small soft submodules and maximal soft submodules. In 2014, Shah and Medhit [15] investigated primary decomposition in a soft ring and a soft module. In 2018, Onar et al. [16] studied vague soft modules.

In 2017, Yucel and Acar [17] defined essential soft submodule and complement of a soft submodule, and obtained some basic properties of these soft submodules.

The main purpose of this paper is to introduce the concept of the socle of a soft submodule and another definition of essential soft submodules which is a little different from that given 
in [17], to investigate basic properties of essential soft submodule and the socle of a soft module, and to give several equivalent definitions of essential soft submodule, the socle and the radical of soft modules, respectively.

\section{Preliminary}

Definition 1 ( [1]). Let $U$ be an initial universe set, $E$ be a set of parameters, $P(U)$ be the power set of $U$ and $A \subseteq E$. An ordered pair $(F, A)$ is called a soft set over $U$, where $F$ is a set valued mapping given by

$$
F: A \rightarrow P(U)
$$

Definition 2 ( [2]). Let $(F, A)$ and $(G, B)$ be soft sets over a common universe $U$. If $B \subseteq A$ and $G(x) \subseteq F(x)$ for every $x \in B$, then we say that $(G, B)$ is a soft subset of $(F, A)$, denoted by $(G, B) \tilde{\subseteq}(F, A)$.

Example 3. Let $(F, A)$ be a soft set over $U$ and $B \subseteq A$. Then $\left(\left.F\right|_{B}, B\right) \tilde{\subseteq}(F, A)$, where $\left.F\right|_{B}$ is the restriction of $F$ on $B$.

Definition 4 ( [14]). Let $(F, A)$ and $(G, B)$ be soft sets over a common universe $U$, and $A \cap B \neq \Phi$. The restricted intersection of $(F, A)$ and $(G, B)$ is denoted by $(F, A) \cap(G, B)$, and is defined to be $(F, A) \cap(G, B)=(H, A \cap B)$, where $H(x)=$ $F(x) \cap G(x)$ for all $x \in A \cap B$.

Throughout this paper, $R$ is an associative ring with identity and all modules are left $R$-modules, unless otherwise stated. Let $M$ and $N$ be $R$-modules. By $N \leq M$, we mean that $N$ is a submodule of $M$.

Now we introduce soft module.

Definition 5 ( [7]). Let $M$ be an $R$-module and $(F, A)$ be a soft set over $M$. We say that $(F, A)$ is a soft module over $M$ if $F(a) \leq M$ for all $a \in A$.

Definition 6 ( [7] ). Let $(F, A)$ and $(G, B)$ be soft modules over $M$. $(G, B)$ is said to be a soft submodule of $(F, A)$, denoted by $(G, B) \tilde{\leq}(F, A)$, if $B \subseteq A$ and $G(b) \leq F(b)$ for all $b \in B$. If $(G, B) \tilde{\leq}(F, A)$ and $(F, A) \tilde{\leq}(G, B)$, then we say two soft modules $(G, B)$ and $(F, A)$ are soft equal and write $(G, B)=$ $(F, A)$.

Example 7. Let $(F, A)$ be a soft module over $M$ and $B \subseteq A$. Then $\left(\left.F\right|_{B}, B\right) \tilde{\leq}(F, A)$, where $\left.F\right|_{B}$ is the restriction of $F$ on $B$.
Definition $8([8])$. A soft module $(F, A)$ over $M$ is called whole if $F(a)=M$ for every $a \in A$. A soft module $(F, A)$ over $M$ is called trivial if $F(a)=0$, for every $a \in A$, denoted by $(F, A)=0$.

Directly from this definition we see that every module $M$ and set $A$ give a whole soft module and a trivial soft module.

Now we define minimal soft submodule.

Definition 9. Let $(G, B) \tilde{\leq}(F, A)$ be soft modules over $M$. $(G, B)$ is said to be a minimal soft submodule of $(F, A)$, if $G(b)$ is a minimal submodule of $F(b)$ for all $b \in B$.

Example 10. Let $n$ be a big integer and $n=p_{1}^{e_{1}} \cdots p_{t}^{e_{t}}$ be its prime decomposition with $e_{i}>0$. Take $M=\mathbb{Z}_{n}$ as a $\mathbb{Z}_{\text {- }}$ module. Let $A=\mathbb{Z}, B=\left\{n / p_{i} \mid i=1, \ldots, t\right\}, F(a)=\mathbb{Z}_{n}$ for $a \in A$ and $G(b)=b \mathbb{Z}_{n}$ for $b \in B$. Then $G(b)$ is a minimal submodule of $F(b)$ for each $b \in B$, so that $(G, B)$ is a minimal soft submodule of $(F, A)$.

We use the following definitions of several special kind of soft submodules from the references.

Definition 11 ([14]). Let $(G, B) \tilde{\leq}(F, A)$ be soft modules over $M .(G, B)$ is said to be a maximal soft submodule of $(F, A)$, if $G(b)$ is a maximal submodule of $F(b)$ for all $b \in B$.

Example 12. Let $n$ be a big integer and $n=p_{1}^{e_{1}} \cdots p_{t}^{e_{t}}$ be its prime decomposition with $e_{i}>0$. Take $M=\mathbb{Z}_{n}$ as a $\mathbb{Z}$-module. Let $A=\mathbb{Z}, B=\left\{p_{i} \mid i=1, \ldots, t\right\}, F(a)=\mathbb{Z}_{n}$ for $a \in A$ and $G(b)=b \mathbb{Z}_{n}$ for $b \in B$. Then $G(b)$ is a maximal submodule of $F(b)$ for each $b \in B$, so that $(G, B)$ is a maximal soft submodule of $(F, A)$.

Definition 13 ([14]). Let $(G, B) \tilde{\leq}(F, A)$ be soft modules over $M$. $(G, B)$ is said to be a small (superfluous) soft submodule of $(F, A)$, if $G(b) \ll F(b)$ for all $b \in B$, denoted by $(G, B) \widetilde{\ll}(F, A)$.

Definition 14 ( [14]). Let $(F, A)$ be a soft module over $M$, and $\left\{\left(F_{i}, A_{i}\right)\right\}_{i \in I}$ be a family of soft submodules of $(F, A)$, where $I$ is a nonempty set. The sum of the soft submodules $\left\{\left(F_{i}, A_{i}\right)\right\}_{i \in I}$ of $(F, A)$ is defined to be

$$
\tilde{\Sigma}_{i \in I}\left(F_{i}, A_{i}\right)=\left(H, \cup_{i \in I} A_{i}\right)
$$

where $H(a)=\Sigma_{i \in I(a)} F_{i}(a), I(a)=\left\{i \in I \mid a \in A_{i}\right\}$ for $a \in \cup_{i \in I} A_{i}$. Particularly, for soft submodules $\left(F_{1}, A_{1}\right)$, $\ldots,\left(F_{n}, A_{n}\right)$ of $(F, A)$, the sum of $\left(F_{1}, A_{1}\right), \ldots,\left(F_{n}, A_{n}\right)$ is denoted by $\left(F_{1}, A_{1}\right) \tilde{+} \cdots \tilde{+}\left(F_{n}, A_{n}\right)$. 
Lemma 15 ([14]). Let $(F, A)$ be a soft module over $M$, and $\left\{\left(F_{i}, A_{i}\right)\right\}_{i \in I}$ be a family of soft submodules of $(F, A)$. Then $\tilde{\Sigma}_{i \in I}\left(F_{i}, A_{i}\right)$ is a soft submodule of $(F, A)$, and $\left(F_{i}, A_{i}\right) \tilde{\leq}$ $\tilde{\Sigma}_{i \in I}\left(F_{i}, A_{i}\right)$.

Proposition 16 ( [14]). Let $(G, B) \tilde{\leq}(F, A)$ be soft modules over $M$. Then

$$
(G, B) \tilde{+}(F, A)=(F, A) .
$$

Definition 17 ( [7]). Let $M$ and $N$ be $R$-modules, $(F, A)$ and $(G, B)$ be soft modules over $M$ and $N$ respectively. The ordered pair $(f, g)$ is called a soft homomorphism, in case $f: M \rightarrow N$ is a homomorphism of modules, $g: A \rightarrow B$ is a mapping, such that $f(F(a))=G(g(a))$ for all $a \in A$. In this case, we write $(f, g):(F, A) \rightarrow(G, B)$.

\section{Essential Soft Submodules}

Essential submodule plays an important role in module theory. Recall the definition of essential submodule. Let $M$ be an $R$ module, $K$ be a submodule of $M . K$ is essential in $M$, in case $K \cap L \neq 0$ for every nontrivial submodule $L$ of $M$, denoted by $K \unlhd M$. For the basic properties of essential submodules see [18-20]. In 2017, Yucel and Acar [17] introduced a definition of essential soft submodules and investigated the basic properties of essential soft submodules.

Definition 18 ( [17]). Let $(F, A)$ be a soft module over $M$. A nontrivial soft submodule $(G, B)$ of $(F, A)$ is called essential soft submodule in $(F, A)$, denoted by $(G, B) \tilde{\leq}_{e}(F, A)$, if $(G, B) \cap(T, C)$ is nontrivial for every nontrivial submodule $(T, C)$ of $(F, A)$ such that $B \cap C \neq \emptyset$.

In this section, we give another definition of essential soft submodules, then give an equivalent condition of such defined essential submodules and study the property of essential submodule under soft homomorphism.

Definition 19. Let $(F, A)$ be a soft module over $M$. A nontrivial soft submodule $(G, B)$ of $(F, A)$ is called essential soft submodule in $(F, A)$, denoted by $(G, B) \tilde{\unlhd}(F, A)$, if $(G, B)$ ก $(T, C)$ is nontrivial for every nontrivial soft submodule of $(F, A)$.

The following theorem provides an equivalent definition of essential soft module.

Theorem 20. Let $(G, B) \tilde{\leq}(F, A)$ be soft modules over $M$, then $(G, B) \tilde{\unlhd}(F, A)$ if and only if $A=B$ and $G(a) \unlhd F(a)$ for each $a \in A$.
Proof. Suppose $A=B$ and $G(a) \unlhd F(a)$ for each $a \in A$. If there is a soft submodule $(H, C)$ of $(F, A)$ such that $(G, A)$ ก $(H, C)=0$. Then $G(c) \cap H(c)=0$ for $c \in C \subseteq A$. While $G(c)$ is essential in $F(c)$, so $H(c)=0$ for any $c \in C$, i.e., $(H, C)=0$.

Conversely, suppose $(G, B) \tilde{\unlhd}(F, A)$. Assume $B \subsetneq A$, then $A-B \neq \emptyset$. Let $(T, A)$ be a soft submodule of $(F, A)$ such that

$$
T(a)= \begin{cases}F(a), & a \in A-B, \\ 0, & a \in B,\end{cases}
$$

then $(T, A)$ is a nontrivial soft submodule of $(F, A)$ and $(G, B) \cap$ $(T, A)$ is trivial, a contradiction. Hence $A=B$. For $a \in A$, if there is some $K \leq F(a)$ such that $K \cap G(a)=0$. Take

$$
C=\{a\}, T(a)=K \text {. }
$$

Then $C \subseteq A,(T, C) \tilde{\leq}(F, A)$ and $(T, C)$ ก $(G, A)=0$, which implies $(T, C)=0$. In particular, $K=T(a)=0$. Therefore $G(a) \unlhd F(a)$ for each $a \in A$.

Every soft module has an essential soft submodule, since every module has an essential submodule. A trivial example of essential soft submodule is $(F, A) \tilde{\unlhd}(F, A)$ for any soft module $(F, A)$. For nontrivial example see the following.

Example 21. Take $\mathbb{Z}$ as a $\mathbb{Z}$-module. Let $(F, \mathbb{N})$ be a whole soft module over $\mathbb{Z}$, i.e., $F(n)=\mathbb{Z}$ for all $n \in \mathbb{N}$. Let $(G, \mathbb{N})$ be a soft module over $\mathbb{Z}$ with $G(n)=n \mathbb{Z}, n \in \mathbb{N}$. Note that $n \mathbb{Z} \unlhd \mathbb{Z}$, so $(G, \mathbb{N}) \tilde{\unlhd}(F, \mathbb{N})$ by Theorem 20

As a corollary of Theorem 20, we give another equivalent definitions for essential soft submodule.

Corollary 22. Let $(G, B) \tilde{\leq}(F, A)$ be soft modules over ${ }_{R} M$. Then $(G, B) \tilde{\unlhd}(F, A)$ if and only if $A=B$ and for each $a \in A$, $0 \neq x \in F(a)$, there exists an $r \in R$ such that $0 \neq r x \in G(a)$.

Proof. By Theorem 20, $(G, B) \tilde{\unlhd}(F, A)$ if and only if $A=B$ and $G(a) \unlhd F(a)$ for any $a \in A$, which is equivalent to say that, for any $a \in A$ and $0 \neq x \in F(a)$, there is an $r \in R$ such that $0 \neq r x \in G(a)$ [18, Lemma 5.19].

Definition 23. Let $(F, A)$ and $(G, B)$ be soft modules over $M$ and $N$, respectively, $(H, C) \tilde{\leq}(F, A)$, and $(f, g):(F, A) \rightarrow$ $(G, B)$ be a soft homomorphism. Define $H^{\prime}(c)=f(H(c))$, $c \in C$. Then $\left(H^{\prime}, C\right)$ is a soft module over $N$, called the image of $(H, C)$ under $(f, g)$, denoted by $(f, g)(H, C)$. Let $(K, D) \tilde{\leq}(G, B)$. For $d \in D$, define $K^{\prime}(d)=f^{\leftarrow}(K(d))$. 
Then $\left(K^{\prime}, D\right)$ is a soft module over $M$, called the preimage of $(K, D)$ under $(f, g)$, denoted by $(f, g)^{\leftarrow}(K, D)$.

Directly from this definition we get the following.

Proposition 24. Let $(f, g):(F, A) \rightarrow(G, B)$ be a soft homomorphism. Then

(1) for any $\left(H_{1}, C_{1}\right) \tilde{\leq}\left(H_{2}, C_{2}\right) \tilde{\leq}(F, A)$,

$$
(f, g)\left(H_{1}, C_{1}\right) \tilde{\leq}(f, g)\left(H_{2}, C_{2}\right)
$$

(2) for any $\left(K_{1}, D_{1}\right) \tilde{\leq}\left(K_{2}, D_{2}\right) \tilde{\leq}(G, B)$,

$$
(f, g)^{\leftarrow}\left(K_{1}, D_{1}\right) \tilde{\leq}(f, g)^{\leftarrow}\left(K_{2}, D_{2}\right)
$$

(3) for any $\left(K_{1}, D_{1}\right) \tilde{\unlhd}\left(K_{2}, D_{2}\right) \tilde{\leq}(G, B)$,

$$
(f, g)^{\leftarrow}\left(K_{1}, D_{1}\right) \tilde{\unlhd}(f, g)^{\leftarrow}\left(K_{2}, D_{2}\right) .
$$

Proof. If $\left(H_{1}, C_{1}\right) \tilde{\leq}\left(H_{2}, C_{2}\right)$, then $H_{1}(c) \leq H_{2}(c)$ for any $c \in C_{1}$. Hence $f\left(H_{1}(c)\right) \leq f\left(H_{2}(c)\right)$. If $\left(K_{1}, D_{1}\right) \tilde{\leq}\left(K_{2}, D_{2}\right)$, then we have $K_{1}(d) \leq K_{2}(d)$ for any $d \in D_{1}$. Hence $f^{\leftarrow}\left(K_{1}(d)\right)$ $\leq f^{\leftarrow}\left(K_{2}(d)\right)$. Furthermore, suppose $\left(K_{1}, D_{1}\right) \tilde{\unlhd}\left(K_{2}, D_{2}\right)$, then $K_{1}(d) \unlhd K_{2}(d)$ for any $d \in D_{1}$ which implies $f^{\leftarrow}\left(K_{1}(d)\right)$ $\unlhd f^{\leftarrow}\left(K_{2}(d)\right)$.

\section{The Socle of Soft Modules}

In this section, we introduce the definition of the socle of soft modules and investigate its basic properties. We also give two equivalent expressions of the socle of soft modules.

Definition 25. Let $(F, A)$ be a soft module over $M$. The socle of $(F, A)$ is defined to be a soft module $(S, A)$ over $M$, such that $S(a)=\operatorname{Soc} F(a)$ for all $a \in A$, denoted by $(S, A)=$ $\operatorname{Soc}_{M}(F, A)$.

Note that the socle of $\mathbb{Z}$ is zero, so the socle of a whole soft module $(F, A)$ over the regular module $\mathbb{Z} \mathbb{Z}$ is trivial. Next we give some nontrivial examples.

Example 26. Let $n$ be a big integer and $n=p_{1}^{e_{1}} \cdots p_{t}^{e_{t}}$ be its prime decomposition with $e_{i}>0$. Take $M=\mathbb{Z}_{n}$ as a $\mathbb{Z}$ module. Let $A=\mathbb{N}$, and $F(a)=\mathbb{Z}_{n}$ for $a \in A$. Then $(F, A)$ is a soft module over $\mathbb{Z}_{n}$ and

$$
\operatorname{Soc} F(a)=\operatorname{Soc} \mathbb{Z}_{n}=\sum_{p_{i} \mid n} \frac{n}{p_{i}} \mathbb{Z}_{n} \cong \oplus_{p_{i} \mid a} \mathbb{Z}_{p_{i}} .
$$

Therefore, $\operatorname{Soc}_{M}(F, A)=(S, A)$ and $S(a) \cong \oplus_{p_{i} \mid a} \mathbb{Z}_{p_{i}}$.
Directly from Definition 25 we get $\operatorname{Soc}_{M}(F, A) \tilde{\leq}(F, A)$. For a module $M, \operatorname{Soc} M$ is the largest semisimple submodule. We will show that, the socle of soft modules is also the largest semisimple soft submodule of $(F, A)$. Now we define a simple (semisimple) soft submodule.

Definition 27. Let $(F, A)$ be a soft module over $M$. A soft submodule $(G, B)$ of $(F, A)$ is said to be simple (semisimple), if $F(a)$ is a simple (semisimple) submodule of $M$ for all $a \in A$.

Example 28. Let $n$ be an integer. Take $M=\mathbb{Z}_{n}$ as a $\mathbb{Z}$-module. Let $A=\{a>0 \mid n / a$ is prime $\}$, and $F(a)=a \mathbb{Z}_{n}$ for $a \in A$. Then $F(a) \cong \mathbb{Z}_{p}$ where $p=n / a,(F, A)$ is a simple soft module over $\mathbb{Z}_{n}$ and $\operatorname{Soc} F(a)=\operatorname{Soc} a \mathbb{Z}_{n}=a \mathbb{Z}_{n}=F(a)$. Therefore, $\operatorname{Soc}_{M}(F, A)=(F, A)$.

Example 29. Let $n$ be a square free integer. Take $M=\mathbb{Z}_{n}$ as a $\mathbb{Z}$-module. Then $M=\mathbb{Z}_{p_{1}} \oplus \cdots \oplus \mathbb{Z}_{p_{t}}$ is semisimple. Let $A=\{a>0|a| n\}$, and $F(a)=a \mathbb{Z}_{n}$ for $a \in A$. Then $(F, A)$ is a semisimple soft module over $\mathbb{Z}_{n}$ and $\operatorname{Soc} F(a)=$ Soc $a \mathbb{Z}_{n}=a \mathbb{Z}_{n}=F(a)$. Therefore, $\operatorname{Soc}_{M}(F, A)=(F, A)$.

Proposition 30. Let $(F, A)$ be a soft module over $M$. Then $\operatorname{Soc}_{M}(F, A)$ is the largest semisimple soft submodule of $(F, A)$.

Proof. Note that $\operatorname{Soc} F(a)$ is the largest semisimple submodule of $F(a)$, so $\operatorname{Soc}_{M}(F, A)$ is semisimple. Let $(G, B) \tilde{\leq}(F, A)$ be semisimple. Then $G(b) \leq S o c F(b)$ for all $b \in B$, hence $(G, B) \tilde{\leq} \operatorname{Soc}(F, A)$.

Proposition 31. Let $(F, A)$ be a soft module over $M,(G, B) \tilde{\leq}$ $(F, A)$. Then,

$$
\operatorname{Soc}_{M}(G, B)=(G, B) \text { ก } \operatorname{Soc}_{M}(F, A) .
$$

Particularly, $\operatorname{Soc}_{M}\left(\operatorname{Soc}_{M}(F, A)\right)=\operatorname{Soc}_{M}(F, A)$.

Proof. Since $(G, B) \tilde{\leq}(F, A)$, then $G(b) \leq F(b)$ for all $b \in$ $B$. [18, Corollary 9.9] implies $\operatorname{Soc} G(b) \leq S o c F(b)$ for all $b \in B$, hence $\operatorname{Soc}_{M}(G, B) \tilde{\leq} \operatorname{Soc}_{M}(F, A)$ and then the first conclusion follows. Taking $(G, B)=\operatorname{Soc}_{M}(F, A)$ gives the second equation.

Proposition 32. Let $(F, A)$ be a soft module over $M$. Then $\operatorname{Soc}_{M}(F, A) \tilde{\unlhd}(F, A)$ if and only if each nontrivial soft submodule of $(F, A)$ contains a minimal soft submodule.

Proof. Suppose $\operatorname{Soc}_{M}(F, A) \tilde{\unlhd}(F, A)$. Then $\operatorname{Soc} F(a) \unlhd F(a)$ for all $a \in A$. Let $(H, B)$ be a nontrivial soft submodule of $(F, A)$, then there is some $b \in B \subseteq A$ such that $H(b)$ is a nonzero submodule of $F(b)$. It follows from [18, Corollary 
9.10] that $H(b)$ contains a minimal submodule. Let $L_{b}$ be a minimal submodule of $H(b)$. Define $(T, C)$ to be the nontrivial soft submodule of $(H, B)$ with

$$
C=\{b\}, T(b)=L_{b} .
$$

Then $(T, C)$ is a minimal soft submodule of $(H, B)$.

Conversely, suppose each nonzero soft submodule of $(F, A)$ contains a minimal soft submodule. For $a \in A$, let $K_{a}$ be any nonzero submodule of $F(a)$. Define $\left(G_{K_{a}}, B_{K_{a}}\right)$ to be the nonzero soft submodule of $(F, A)$ with

$$
B_{K_{a}}=\{a\}, G_{K_{a}}(a)=K_{a} .
$$

Then $\left(G_{K_{a}}, B_{K_{a}}\right)$ is a nontrivial soft submodule of $(F, A)$. Let $(T, C)$ be a minimal soft submodule of $\left(G_{K_{a}}, B_{K_{a}}\right)$. Then $C=$ $B_{K_{a}}=\{a\}$ and $T(a)$ is a minimal submodule of $G_{K_{a}}(a)=$ $K_{a}$, i.e., a minimal submodule of $F(a)$. That is to say, every nonzero submodule of $F(a)$ contains minimal submodule. It follows from [18. Corollary 9.10] that $\operatorname{Soc} F(a) \unlhd F(a)$ for all $a \in A$, hence $\operatorname{Soc}_{M}(F, A) \tilde{\unlhd}(F, A)$.

Proposition 33. Let $(F, A)$ and $(G, B)$ be soft modules over $M$ and $N$, respectively, $(f, g):(F, A) \rightarrow(G, B)$ be a soft homomorphism. Then

$$
(f, g)\left(\operatorname{Soc}_{M}(F, A)\right) \tilde{\leq} \operatorname{Soc}_{N}(G \circ g, A) .
$$

Proof. Since $(f, g)$ is a soft homomorphism, then $f(F(a))=$ $G(g(a))$ for all $a \in A$. Applying [18. Proposition 9.8] to the homomorphism $\left.f\right|_{F(a)}: F(a) \rightarrow G(g(a))$ gives $f(\operatorname{Soc} F(a)) \leq$ $\operatorname{Soc} G(g(a))$. Therefore $(f, g)\left(\operatorname{Soc}_{M}(F, A)\right) \tilde{\leq} \operatorname{Soc}_{N}(G \circ g, A)$.

We find two equivalent definitions of the socle of soft module.

Theorem 34. Let $(F, A)$ be a soft module over $M$. Then

$$
\begin{aligned}
\operatorname{Soc}_{M}(F, A) & =\cap\{(G, A) \mid(G, A) \text { is essential in }(F, A)\} \\
& =\tilde{\Sigma}\{(G, B) \mid(G, B) \text { is minimal in }(F, A)\} .
\end{aligned}
$$

\section{Proof. Denote}

$$
\begin{aligned}
& (S, A)=\operatorname{Soc}_{M}(F, A), \\
& (H, A)=\text { ก }\{(G, A) \mid(G, A) \text { is essential in }(F, A)\} .
\end{aligned}
$$

Then $H(a)=\cap_{(G, A) \tilde{\unlhd}(F, A)} G(a)$ for each $a \in A$. That is to say, $H(a)$ is an intersection of some essential submodules of $F(a)$. By Definition 25] and [18, Proposrion 9.7], $S(a)=$
Soc $F(a)$ is the intersection of all of the essential submodules of $F(a)$, then $S(a) \leq H(a)$. Hence $(S, A) \tilde{\leq}(H, A)$.

Conversely, for $a_{0} \in A$, let $L_{a_{0}}$ be any essential submodule of $F\left(a_{0}\right)$. Define

$$
G_{L_{a_{0}}}(a)= \begin{cases}L_{a_{0}}, & a=a_{0}, \\ F(a), & a \neq a_{0}, a \in A .\end{cases}
$$

Then $\left(G_{L_{a_{0}}}, A\right)$ is an essential soft submodule of $(F, A)$. So

$$
(H, A)=\cap\{(G, A) \mid(G, A) \tilde{\unlhd}(F, A)\} \tilde{\leq}\left(G_{L_{a_{0}}}, A\right)
$$

for each $a_{0} \in A$ and $L_{a_{0}}$ essential in $F\left(a_{0}\right)$. Hence

$$
(H, A) \tilde{\leq} \cap\left\{\left(G_{L_{a_{0}}}, A\right) \mid L_{a_{0}} \unlhd F\left(a_{0}\right), a_{0} \in A\right\} .
$$

Notice that for each $a_{0} \in A, L_{a_{0}}$ go through all of the essential soft submodule of $F(a)$, then by [18, Proposition 9.7], $\cap_{L_{a_{0}} \unlhd F\left(a_{0}\right)} L_{a_{0}}=\operatorname{Soc} F\left(a_{0}\right)$. Then (1) reads $(H, A) \tilde{\leq}(S, A)$.

To proof the second equation, denote

$$
(K, A)=\tilde{\Sigma}\{(G, B) \mid(G, B) \text { is minimal in }(F, A)\} .
$$

Then, $K(a)=\Sigma_{a \in B} G(a)$, where $(G, B)$ goes through all the minimal soft submodules of $(F, A)$ with $a \in B$. That is to say, $K(a)$ is a sum of some minimal submodules of $F(a)$. Note that $S(a)=\operatorname{Soc} F(a)$ is the sum of all of the minimal submodules of $F(a)$ [18, Proposition 9.7], then $K(a) \leq S(a)$ for all $a \in A$. Therefore, $(K, A) \tilde{\leq}(S, A)$.

Conversely, for $a \in A$, if $S_{a}$ is a minimal submodule of $F(a)$, define

$$
B_{S_{a}}=\{a\}, G_{S_{a}}(a)=S_{a} .
$$

Then $\left(G_{S_{a}}, B_{S_{a}}\right)$ is a minimal soft submodule of $(F, A)$ and

$$
\begin{aligned}
\left(G_{S_{a}}, B_{S_{a}}\right) & \tilde{\leq} \tilde{\Sigma}\{(G, B) \mid(G, B) \text { is minimal in }(F, A)\} \\
& =(K, A) .
\end{aligned}
$$

Hence

$$
\begin{aligned}
(S, A) & =\tilde{\Sigma}\left\{\left(G_{S_{a}}, B_{S_{a}}\right) \mid S_{a} \text { is minimal in } F(a), a \in A\right\} \\
& \tilde{\leq}(K, A)
\end{aligned}
$$

where for the first equation we use the fact $\Sigma\left\{S_{a} \mid S_{a}\right.$ is minimal in $F(a)\}=\operatorname{Soc} F(a)$ for all $a \in A[18$. Proposition 9.7]. 


\section{The Radical of Soft Modules}

In 2013, Turkmen and Pancar [14] defined the radical of soft module.

Definition 35 ( $[14])$. Let $(F, A)$ be a soft module over $M$. The radical of $(F, A)$ is defined to be $(R, A)$, where $R(a)=$ $\operatorname{Rad} F(a)$ for all $a \in A$, and denoted by $(R, A)=\operatorname{Rad}_{M}(F, A)$.

Now we compute the soft radicals of the examples we discuss in last section.

Example 36. Let $n$ be a big integer and $n=p_{1}^{e_{1}} \cdots p_{t}^{e_{t}}$ be its prime decomposition with $e_{i}>0$. Take $M=\mathbb{Z}_{n}$ as a $\mathbb{Z}$ module. Let $A=\mathbb{N}$, and $F(a)=\mathbb{Z}_{n}$ for $a \in A$. Then $(F, A)$ is a soft module over $\mathbb{Z}_{n}$ and $\operatorname{RadF}(a)=\operatorname{Rad} \mathbb{Z}_{n}=\cap_{p_{i} \mid n} p_{i} \mathbb{Z}_{n}=$ $p_{1} \cdots p_{t} \mathbb{Z}_{n} \cong \oplus_{p_{i} \mid a} \mathbb{Z}_{p_{i}^{e_{i}-1}}$. Therefore, $\operatorname{Rad}_{M}(F, A)=(R, A)$ and $R(a) \cong \oplus_{p_{i} \mid a} \mathbb{Z}_{p_{i}^{e_{1}-1}}$.

Example 37. Let $n$ be an integer. Take $M=\mathbb{Z}_{n}$ as a $\mathbb{Z}_{\text {- }}$ module. Let $A=\{a>0 \mid n / a$ is prime $\}$, and $F(a)=a \mathbb{Z}_{n}$ for $a \in A$. Then $\operatorname{Rad} F(a)=\operatorname{Rad} a \mathbb{Z}_{n}=0$. Therefore, $\operatorname{Rad}_{M}(F, A)=0$.

Turkmen and Pancar ( [14, Theorem 9, 10]) discussed the properties of the radical of soft module and proved

$$
\begin{aligned}
& \tilde{\Sigma}\{(G, B) \mid(G, B) \tilde{\ll}(F, A)\} \tilde{\leq} \operatorname{Rad}_{M}(F, A), \\
& \operatorname{Rad}_{M}(F, A) \tilde{\leq} \cap\{(G, A) \mid(G, A) \text { is maximal in }(F, A)\} .
\end{aligned}
$$

We find that these two relations actually induce two equivalent definitions of the radical of soft module.

Theorem 38. Let $(F, A)$ be a soft module over $M$. Then

$$
\begin{aligned}
\operatorname{Rad}_{M}(F, A) & =\tilde{\Sigma}\{(G, B) \mid(G, B) \text { is small in }(F, A)\} \\
& =\cap\{(G, A) \mid(G, A) \text { is maximal in }(F, A)\} .
\end{aligned}
$$

Proof. For $a \in A$, let $K_{a}$ be any small soft submodule of $F(a)$. Define

$$
B_{K_{a}}=\{a\}, G_{K_{a}}(a)=K_{a} .
$$

Then $\left(G_{K_{a}}, B_{K_{a}}\right) \tilde{\ll}(F, A)$ and Lemma 15 implies

$$
\left(G_{K_{a}}, B_{K_{a}}\right) \tilde{\Sigma} \tilde{\Sigma}\{(G, B) \mid(G, B) \tilde{\ll}(F, A)\}
$$

so that

$$
\begin{aligned}
& \tilde{\Sigma}\left\{\left(G_{K_{a}}, B_{K_{a}}\right) \mid K_{a} \ll F(a), a \in A\right\} \\
& \tilde{\leq} \tilde{\Sigma}\{(G, B) \mid(G, B) \tilde{\ll}(F, A)\} .
\end{aligned}
$$

Notice that for each $a \in A, K_{a}$ go through all of the small soft submodule of $F(a)$, then by [18, Proposition 9.13], $\Sigma\left\{K_{a} \mid\right.$ $\left.K_{a} \ll F(a)\right\}=\operatorname{Rad} F(a)$ for each $a \in A$. Hence equation (3) reads

$$
\operatorname{Rad}_{M}(F, A) \tilde{\leq} \tilde{\Sigma}\{(G, B) \mid(G, B) \text { is small in }(F, A)\},
$$

and then [14, Theorem 9] implies the first equation.

To proof the second equation, fix a maximal submodule $M_{a} \leq F(a)$ for each $a \in A$. For $a_{0} \in A$, if $L_{a_{0}}$ is a maximal submodule of $F\left(a_{0}\right)$, define

$$
G_{L_{a_{0}}}(a)= \begin{cases}L_{a_{0}}, & a=a_{0}, \\ M_{a}, & a \neq a_{0}, a \in A .\end{cases}
$$

Then $\left(G_{L_{a_{0}}}, A\right)$ is a maximal soft submodule of $(F, A)$. Hence

$$
\text { ก }\{(G, A) \mid(G, A) \text { is maximal in }(F, A)\} \stackrel{\tilde{\leq}}{\leq}\left(G_{L_{a_{0}}}, A\right)
$$

for each $a_{0} \in A$ and $L_{a_{0}}$ maximal in $F\left(a_{0}\right)$, and then

$$
\begin{aligned}
& \text { ก }\{(G, A) \mid(G, A) \tilde{\leq}(F, A) \text { maximal }\} \\
& \tilde{\leq} \cap\left\{\left(G_{L_{a_{0}}}, A\right) \mid L_{a_{0}} \leq F\left(a_{0}\right) \text { maximal, } a_{0} \in A\right\} \\
& =\operatorname{Rad}_{M}(F, A) .
\end{aligned}
$$

Here, for the last equation we use the fact that $\cap\left\{L_{a} \mid L_{a}\right.$ is maximal in $F(a)\}=\operatorname{Rad} F(a)$ for each $a \in A[18$, Proposition 9.13]. Then [14, Theorem 10] implies the second equation.

\section{Conclusion}

In this paper, we defined the socle of a soft module and redefine essential soft submodules which is a little different from [17], investigated the property of essential soft submodule and the socle of a soft module. Further, we gave several equivalent definitions of essential soft submodule, the socle and the radical of soft modules.

\section{Conflict of Interest}

No potential conflict of interest relevant to this article was reported. 


\section{Acknowledgments}

This work is supported by China NNSF (Grant No. 11771176). The authors thanks the referees for reading the manuscript carefully and their helpful comments.

\section{References}

[1] D. Molodtsov, "Soft set theory-first results," Computers \& Mathematics with Applications, vol. 37, no. 4-5, pp. 19-31, 1999. https://doi.org/10.1016/S0898-1221(99)00056-5

[2] P. K. Maji, R. Biswas, and A. R. Roy, "Soft set theory," Computers \& Mathematics with Applications, vol. 45, no. 4-5, pp. 555-562, 2003. https://doi.org/10.1016/S08981221(03)00016-6

[3] H. Aktas and N. Cagman, "Soft sets and soft groups," Information Sciences, vol. 177, no. 13, pp. 2726-2735, 2007. https://doi.org/10.1016/j.ins.2006.12.008

[4] F. Feng, Y. B. Jun, and X. Zhao, "Soft semirings," Computers \& Mathematics with Applications, vol. 56, no. 10, pp. 2621-2628, 2008. https://doi.org/10.1016/j.camwa.2008. 05.011

[5] U. Acar, F. Koyuncu, and B. Tanay, "Soft sets and soft rings," Computers \& Mathematics with Applications, vol. 59, no. 11, pp. 3458-3463, 2010. https://doi.org/10.1016/j. camwa.2010.03.034

[6] M. Irfan Ali, F. Feng, X. Liu, W. K. Min, and M. Shabir, "On some new operations in soft set theory," Computers \& Mathematics with Applications, vol. 57, no. 9, pp. 15471553, 2009. https://doi.org/10.1016/j.camwa.2008.11.009

[7] Q. M. Sun, Z. Zhang, and J. Liu, "Soft sets and soft modules," Rough Sets and Knowledge Technology. Heidelberg: Springer, 2008, pp. 403-409. https://doi.org/10.1007/9783-540-79721-0_56

[8] A. O. Atagun and A. Sezgin, "Soft substructures of rings, fields, and modules," Computers \& Mathematics with Applications, vol. 61, no. 3, pp. 592-601, 2011. https: //doi.org/10.1016/j.camwa.2010.12.005

[9] G. X. Xiao, D. Xiang, and J. Zhan, "Fuzzy soft modules," East Asian Mathematics Journal, vol. 28, no. 1, pp. 1-11, 2012.
[10] C. Gunduz and S. Bayramov, "Intuitionistic fuzzy soft modules," Computers \& Mathematics with Applications, vol. 62 , no. 6, no. 6, pp. 2480-2486, 2011. https://doi.org/ 10.1016/j.camwa.2011.07.036

[11] Y. Yin, H. Li, and Y. B. Jun, "On algebraic structure of intuitionistic fuzzy soft sets," Computers \& Mathematics with Applications, vol. 64, no. 9, pp. 2896-2911, 2012. https://doi.org/10.1016/j.camwa.2012.05.004

[12] D. Xiang, "Soft module theory," Proceedings of 2013 10th International Conference on Fuzzy Systems and Knowledge Discovery (FSKD), Shenyang, China, 2013, pp. 103107. https://doi.org/10.1109/FSKD.2013.6816175

[13] T. Y. Ozturk, C. Gunduz, and S. Bayramov, "Inverse and direct systems of soft modules," Annals of Fuzzy Mathematics and Informatics, vol. 5, no. 1, pp. 73-85, 2013.

[14] E. Turkmen and A. Pancar, "On some new operations in soft module theory," Neural Computing and Applications, vol. 22, no. 6, pp. 1233-1237, 2013. https://doi.org/10. 1007/s00521-012-0893-6

[15] T. Shah and S. Medhit, "Primary decomposition in a soft ring and a soft module," Iranian Journal of Science and Technology, vol. 38, no. 3 (special issue: mathematics), pp. 311-320, 2014.

[16] S. Onar, S. Yavuz, B. A. Ersoy, and K. Hila, "Vague soft modules," Journal of Intelligent and Fuzzy Systems, vol. 34, no. 4, pp. 2597-2609, 2018. https://doi.org/10.3233/ JIFS-17497

[17] C. C. Yucel, U. Acar, "A note on soft modules," Communications Faculty of Sciences University Of AnkaraSeries Al Mathematics And Statistics, vol. 66, no. 1, pp. 66-74, 2017. D O I: https://doi.org/10.1501/Commua1_ 0000000775

[18] F. W. Anderson and K. R. Fuller, Rings and Categories of Modules. New York, NY: Springer, 1992.

[19] T. Y. Lam, Lectures on Modules and Rings. New York, NY: Springer, 1999.

[20] R. Wisbauer, Foundations of Module and Ring Theory: A Handbook for Study and Research. Philadelphia, PA: Gordon and Breach Science Publishers, 1991. 


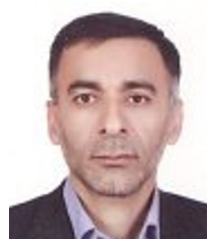

Bijan Davvaz took his B.Sc. degree in Applied Mathematics at Shiraz University, Iran in 1988 and his M.Sc. degree in Pure Mathematics at Tehran University in 1990. In 1998, he received his Ph.D. in Mathematics at Tarbiat Modarres University. He is a member of Editorial Boards of 25 Mathematical journals. He is the author of around 500 research papers, especially on algebraic hyperstructures and fuzzy logic. Moreover, he published seven books in algebra. He was chosen as the best professor (2009) and distinguished researcher (2011) in Iran. He is currently Professor of Mathematics at Yazd University in Iran.

E-mail: bdavvaz@yahoo.com

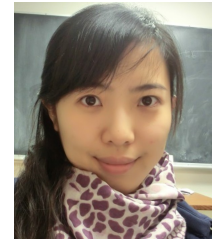

Jing Ma is a professor in the Department of Mathematics at Jilin University, Changchun, China. She received her Ph.D. from Jilin University in 2005. She works on algebra, soft algebra, fuzzy logic and number theory. She is the author of around 30 research papers. She has also published two books on algebra.

E-mail: jma@jlu.edu.cn

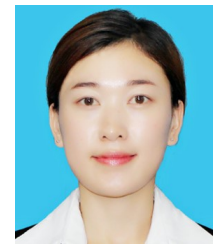

Chengxia Sun is a teacher at United Experimental School of AHSJU and Livon, Changchun, China. She received her M.Sc. from Jilin University in 2016. She works on algebra and soft algebra.

E-mail: cxsun13@emails.jlu.edu.cn 